\title{
Participación, ciudadanía y voto: reflexiones para el Trabajo Social a partir del caso chileno
}

\author{
Nelson Arellano-Escudero \\ Universidad Andres Bello, Chile
}

\author{
Borja Castro-Serrano \\ Universidad Andres Bello, Chile
}

\section{Participación, ciudadanía y voto: reflexiones para el Trabajo Social a partir del caso chileno}

Resumen: Una revisión de antecedentes respecto a la percepción y opiniones acerca del ejercicio democrático y electoral en el modelo político chileno sustenta un análisis que utilizó información de estudios recientes, bases de datos y producción propia. Con un enfoque desde el Trabajo Social se afirma que los significados inscritos en el campo de la intervención social convocan a la profesión a una reflexión que apunte a la comprensión y la consideración del sujeto político contemporáneo como un campo que requiere revisión, análisis y debate para enriquecer el oficio y la disciplina.

Palabras claves: Trabajo Social. Sujeto político. Voto. Ciudadanía. Participación.

\section{Participação, cidadania e voto: reflexões sobre o Serviço Social a partir do caso chileno}

Resumo: Uma revisão sobre as percepções e opiniões acerca do exercício democrático e eleitoral no modelo político chileno comporta análise utilizando informações provenientes de estudos recentes, bases de dados e produção própria. Centrada no Serviço Social, afirma que os significados inscritos no campo da intervenção social requerem uma reflexão que indique a compreensão e a consideração do sujeito político contemporâneo como um campo para revisão, análise e debate a fim de aperfeiçoar a profissão e a disciplina.

Palavras-chave: Trabalho Social. Sujeito político. Voto. Cidadania. Participação.

\section{Participation, Citizenship and the Vote: Reflections for social work based on the Chilean Case}

Abstract: A review of the antecedents concerning the perception and opinions of the exercise of democracy and elections in the Chilean political model sustains an analysis that used information from recent studies, databases and new investigations. With a focus based on social work, the article affirms that the meanings inscribed in the field of social intervention convoke the profession to a reflection that points to the understanding and consideration of the contemporary political subject as a field that requires revision, analysis and debate to enrich the profession and the discipline.

Keywords: Social Work, Political Subject. Vote. Citizenship. Participation. 


\section{El problema de investigación y su recorrido ${ }^{1}$}

Para el Trabajo Social la definición de sujeto de intervención social tiene un componente que implica la comprensión del sujeto político y que, en cierta medida, pudiera estudiarse como ontologías superpuestas o, al menos, con grandes intersecciones conceptuales. Esto implica el desafío de analizar los significados que los sujetos le otorgan a la política y sus instrumentos y, en especial, al arquetipo de la participación ciudadana en las democracias representativas: el

voto. Ahora bien, los datos internacionales sobre democracia, conflicto social, voto y elecciones aparecen con los estudios electorales que se han interesado en la relación entre estructura social y votación en las democracias occidentales (OESCH, 2008). No obstante, la investigación al respecto no ha sido homogénea ni en términos de territorio ni en cuanto a las variables estudiadas (BLAIS, 2008).

A nivel de la región de América Latina, con la complejidad de su historia política, prevalece la opinión de que las democracias han tendido hacia la consolidación (PNUD, 2014), aunque sigan existiendo altos niveles de pobreza y desigualdad (CHIN; CULOTTA, 2014). A nivel local, en Chile se avanzó hacia una inscripción automática y voto voluntario, lo que puede valorarse como un gesto de reconocimiento de la autonomía de la ciudadanía frente al Estado.

La literatura sugiere que se ha logrado una cierta participación política electoral, reconociéndose el voto como un derecho universal ${ }^{2}$, que se ejerce en elecciones transparentes en la mayoría de los países latinoamericanos (PNUD, 2004). Algunos datos empíricos muestran que a nivel de elecciones en América Latina, el nivel de participación es respetable y positiva: "89,3 por ciento de los potenciales votantes están inscritos en los registros electorales, 62,7 por ciento vota y 56,1 por ciento emite un voto válido” (PNUD, 2004, p. 84-85).

Sin embargo, ampliando las fronteras del Trabajo

...un desafío para el Trabajo
Social: resituar el significado y
alcance de la participación
electoral, pues si la democracia
implica una mirada a la
participación y a la ciudadanía
es porque supone una idea de
ser humano en relación con la
construcción social que intenta
poner en juego aquella
ciudadanía integral, ejerciendo
derechos políticos, civiles y
sociales.
Social, pero en esta misma línea de análisis con la que se comienza, es prudente situar la mirada hacia el contexto global y mundial sobre el estado de la democracia y su forma de organización política. Si bien sería el sistema que da estabilidad e igualdad a los mecanismos de participación (de todo tipo), otras apreciaciones alertan de ciertas problemáticas que surgen en las democracias actuales. Lo anterior, parece tener un impacto y un efecto transformador a nivel de la participación, específicamente, en la actividad electoral, generando distanciamientos entre la ciudadanía y las instituciones democráticas. Esto, por cierto, considerando que la democracia electoral no agota la democracia ciudadana (AGAMBEN, 2010). La democracia ciudadana ha sido vista como un campo teórico capaz de relacionar ambas dimensiones, lo electoral y lo ciudadano, mirando sus tensiones y conflictos, así como la relación entre lo social y la democracia. Como resorte de un análisis filosófico político, esta articulación ha sido trabajada de modo crítico, entre otros, por algunos pensadores contemporáneos franceses, que instalan el desafío de incorporar a las democracias actuales elementos de conflictividad y alteridad propios del ser humano que son saludables para su funcionamiento (ABENSOUR, 2007; 1998). Sin embargo, este comportamiento en la actualidad comienza a ser irregular en tanto la participación electoral disminuye porcentualmente al tiempo que se permite la expresión de formas de participación que hasta ahora se habían considerado secundarias o definitivamente de menor valor que el voto. Es en ese contexto que se identifica un desafío para el Trabajo Social: resituar el significado y alcance de la participación electoral, pues si la democracia implica una mirada a la participación y a la ciudadanía es porque supone una idea de ser humano en relación con la construcción social que intenta poner en juego aquella ciudadanía integral, ejerciendo derechos políticos, civiles y sociales. El régimen electoral es un elemento fundamental, pero que no se reduce sólo a las elecciones (PNUD, 2004; ROSANVALLON, 2012).

En el caso de Chile se ha acumulado información acerca de la compleja relación entre democracia, ciudadanía y participación electoral. Según el Observatorio Político Electoral de la Universidad Diego Portales (OBPE, 2012), vemos que el padrón electoral y votaciones cae un 50\% desde 1989 a lo que indica el año 2012. 
Inclusive, la participación electo ral disminuye significativamente con la implementación del voto voluntario. "S i en 2008 votó alrededor del 58 por ciento de todas las personas en edad de votar, en 2012 el porcentaje apenas superó el 41 por ciento" (OBPE, 2012, p. 1). Esta situación abre el debate acerca de las formas de participación, en donde las elecciones son sólo una forma de hacerlo en medio de un abanico de posibilidades, lo que permite repensar la misma noción de participación y su impacto en el concepto de ciudadano, el cual irrumpe como sujeto con más posibilidades sociales y políticas que la mera acción de votar. Si bien Chile tiene niveles de participación relativamente bajos en comparación con la región en ámbitos como los sindicatos, organizaciones sociales, colegios profesionales, centro de padres etc., los datos nos entregan una cierta práctica ciudadana y participativa que convoca más que el acto de votar. Por ejemplo, las estadísticas respecto a la participación de los chilenos en movimientos sociales en los últimos años han aumentado notablemente (PNUD, 2014). Los datos empíricos sostienen que la participación en manifestaciones públicas va en claro aumento, desde el 2008 con 36 por ciento, aumentando el año 2010 a 53 y el 2012 a un 57 (PNUD, 2014). Esta situación sugiere la emergencia de un nuevo sujeto político, investido de voz y argumentos para participar de la democracia chilena desde una posición distinta respecto a las lecturas hegemónicas que le asignaron una performatividad universalista y homogénea, cuando sus características más evidentes son la diversidad y heterogeneidad. Estos datos son coincidentes con los resultados de la encuesta "Ciudadanía hoy"3 (UNAB, 2012), la cual entrega también cifras empíricas del Chile actual que permiten ir sosteniendo el problema recién planteado. Así la escena, parece pertinente que el Trabajo Social asuma el desafío de re-estudiar las formas de participación y ciudadanía examinando con detención los datos de la realidad contemporánea y analizando las características de lo que parece ser un nuevo sujeto político que entra en la escena de la intervención social (MUÑOZ, 2011; 2014).

Así entonces y con el problema circunscrito, este trabajo tiene por objetivo reflexionar sobre los modos de participación y ciudadanía que se ejercen en un contexto democrático y electoral actual para y desde el Trabajo Social en el caso chileno, enmarcado en torno a las siguientes preguntas: ¿implica la baja participación electoral el desafío de resignificar las formas de participación en el Chile actual? ¿Sería este nuevo sujeto político un aliciente para poder resignificar formas tradicionales de transformación e intervención social? Este nuevo escenario, ¿trae implicancias en la comprensión de los contextos de intervención social? Estas serán las preguntas que guiarán el recorrido propuesto. En conjunto, los datos aportados por el caso de estudio y la perspectiva teórica con enfoque desde el Trabajo Social que se analiza, demuestran la necesidad de elaborar una reflexión acerca del sujeto político contemporáneo de modo que la profesión y la disciplina ganen en la densidad conceptual que requiere la comprensión de los procesos de intervención social y, entre otras dimensiones, la alteridad que dota de sentido y esencia al oficio del Trabajo Social.

\section{Trabajo social y votaciones: mundo electoral, participación y sujeto contemporáneo de la intervención}

La comprensión de la calidad o condición de votante de las personas involucradas en la intervención del Trabajo Social parece haber sido omitida durante largo tiempo, según se desprende de las búsquedas preliminares en la literatura disciplinaria en Chile. Esta observación recorre las distintas corrientes teóricas con incidencia en las formas de pensamiento de la profesión y la disciplina. Merece, entonces, una opinión tanto por el estado de situación como por la manera en que se encara la configuración de sujeto que le compete a la intervención social asumir como contraparte. En la mirada local, Arriagada (2013) analiza, en perspectiva sociológica, la relación entre clientelismo político y participación estableciendo claras evidencias de prácticas sociales integradas al imaginario colectivo que en el Trabajo Social, la llamada intervención a nivel de comunidad, podría conocer suficientemente. En las discusiones de trabajadores sociales aparece como un lugar común, es decir, como una frase hecha, que la democracia es más que el ejercicio del derecho al voto. Pero ¿qué significa el voto para el/la sujeto contemporáneo/a?

Desde luego, resulta llamativo que el sujeto imaginado por el Trabajo Social aparezca despolitizado y que la propuesta transformadora de la profesión no haya prestado atención a la condición de votante que, al menos nominalmente, pone en condiciones de igualdad a toda la población que cumpla con los requisitos respectivos. La sorpresa aumenta cuando una somera revisión a la literatura anglosajona muestra que, aunque haya explorado el tema, tampoco ha sido prolífica. Lo atestigua Davis (2010) donde se revisan críticamente los límites de la ciudadanía, cuestionando la exclusión de personas con discapacidad cognitiva en los procesos democráticos. Una aproximación diferente entiende que el vínculo entre bienestar y ciudadanía debe ser explorado buscando una relación entre la estigmatización por recibir beneficios sociales y el desinterés electoral (TOGUCHI et al., 2009). Sin embargo, un amplio compás de espera debe iniciarse cuando acudimos a la perspectiva historiográfica y 
encontramos el rastro que Illanes (2003) siguió de la visitadora social Berta Recabarren, figura también considerada por Vitale (1987) y Kirkwood (1986). La figura de Berta, una de las fundadoras del Partido Cívico Femenino en 1922, debe ser vista a la luz de la declaración expresada en los estatutos, cuyos propósitos consideraban conseguir reformas legales para que las mujeres pudieran tener los derechos negados, entre ellos el voto, tal como fue ocurriendo en muchos países del hemisferio norte entre 1918 y 1920 . No obstante, en el transcurso del siglo 20 el acaecer fue configurando el imaginario de los/las trabajadores/as sociales con una flagrante omisión de la condición política de las personas contraparte en sus labores de intervención social, esto es, segmentando la condición política del sujeto. Este es el estado de situación con el que se inició el análisis de las informaciones disponibles, todas ellas elaboradas en otros ámbitos disciplinarios, y frente a las cuales se contrapuso la encuesta Ciudadanía hoy (UNAB, 2012). De esta última, algunos datos interesantes de analizar se ven en la tabla 1 y 2 , abriendo más luces para el mundo disciplinar del Trabajo Social.

Tabla 1 - "No tiene mucha importancia si voto o no voto, igual soy ciudadano", de acuerdo a Grupo Socioeconómico (GSE) (en porcentaje)

\begin{tabular}{|l|c|c|c|c|c|}
\hline & GSE C1 & GSE C2 & GSE C3 & GSE DE & TOTAL \\
\hline Muy de acuerdo & 12.5 & 19.7 & 38.8 & 28.9 & 100 \\
\hline De acuerdo & 8.2 & 22.9 & 37 & 31.9 & 100 \\
\hline En desacuerdo & 16.5 & 26.3 & 32.1 & 25.1 & 100 \\
\hline Muy desacuerdo & 22.9 & 31.4 & 24.3 & 21.4 & 100 \\
\hline No sabe/no responde & 14.3 & 19 & 33.3 & 33.3 & 100 \\
\hline
\end{tabular}

Fuente: Elaboración propia.

Desde una perspectiva estructural de la organización social los segmentos $\mathrm{AB}$, no considerados en esta encuesta, y el $\mathrm{C} 1$ conforman el cuartil de ingresos más altos de Chile, por lo cual se encuentran fuera del rango de atención del Trabajo Social, así como los segmentos C2 y C3 que representarían los grupos de clase media chilena configurada a mediados del siglo 20. No obstante ello, nuestra atención debiera incorporar los grupos C3, D y E, cuya condición socioeconómica regularmente implica movilidad social vertical asociada a las circunstancias de la economía nacional, es decir, es un segmento de la sociedad que estando en los cuartiles de ingreso inferiores pasan por temporadas de mayor bienestar socioeconómico y otras tantas en situación de pobreza. Por ello resulta muy significativo encontrar la mayor desafección con el voto en los segmentos sociales de menores ingresos y se presta para la conjetura de un eventual imaginario colectivo asociado a unas prácticas sociales contrahegemónicas (GARCÉS, 2002). Esto se refrenda cuando los grupos de menores ingresos conforman más del $60 \%$ de los/as encuestados/as que no consideran una obligación importante de la ciudadanía ejercer el derecho al voto.

Tabla 2 - "Cuáles de las siguientes acciones son para usted parte importante de las obligación de los ciudadanos: votar", de acuerdo a Grupo Socioeconómico (GSE) (en porcentaje)

\begin{tabular}{|l|c|c|c|c|c|}
\hline & GSE C1 & GSE C2 & GSE C3 & GSE DE & TOTAL \\
\hline Sí & 12.3 & 23.5 & 32.6 & 31.6 & 100 \\
\hline No & 12 & 25.3 & 40.3 & 22.4 & 100 \\
\hline
\end{tabular}

Fuente: Elaboración propia

Sin embargo, la ruta de análisis no debiera seguir la huella simplista de la crisis de participación ni la asociación mecánica del desafecto por el voto con riesgos para la gobernabilidad. El cuadro podría ser aún más complejo dados los procesos históricos de continuidad y cambio desde la era colonial que se han proyectado en el llamado tiempo republicano (ILLANES, 2003; GREZ, 2010). Estos factores de análisis son cruciales para la configuración de una propuesta de Trabajo Social crítico y los diseños de las prácticas de intervención social. 
En un paso más allá de los datos, este análisis recién descrito, pretende contribuir a la comprensión de los significados de la ciudadanía contemporánea explorando una trama conceptual que permita interpretar las posibilidades de sujeto político que el ejercicio profesional del Trabajo Social podría encontrar en el Chile actual. Ahora bien, como se ha dicho y se sabe, la relación entre participación y mundo electoral ha sido preferentemente materia de análisis por parte de disciplinas como la sociología y la ciencia política. Así, los referentes de la sociología de la acción de Alain Touraine han tenido profunda repercusión (ANGELCOS, 2011), explorando eventuales respuestas a una cierta situación conceptualizada como el apoliticismo (BAÑO, 1995) que alimentó de manera insistente la imagen de una población desafectada por la tarea electoralista (CANTILLANA, 2009; CARLIN, 2006).

La preocupación académica por el estado de situación electoral en Chile se puede visualizar en la creación en el año 2004 del Observatorio Político Electoral (OBPE) de la Universidad Diego Portales (UDP). La evidencia empírica se puede sintetizar en la impactante disminución de electores/as desde 1989, cuando se superó el 80\% de participación de las personas en edad de votar, hasta 2013 con poco más del $40 \%$. Carlin (2006) aduce tres explicaciones no excluyentes: la falta de apoyo político; despolitización; y un cambio generacional en la cultura política. Esta propuesta podría considerarse una muestra típica de los análisis en los que, en ocasiones, pueden integrarse planteamientos de la filosofía política, como los de Nancy Fraser y Ernesto Laclau (ANGELCOS, 2011).

La búsqueda de explicaciones ha apuntado de preferencia a la búsqueda estadística de relaciones entre variables estructurales: pobreza, educación, edad, entre otras, generando una abundante literatura en participación electoral (CONTRERAS; MORALES, 2014). Y uno de los aspectos de interés para el Trabajo Social es justamente aquel conceptualizado como sesgo de clase, en tanto las elecciones chilenas han ido afianzando la constatación de que una mayor proporción de la población con mayores ingresos acuden a votar, mientras que las poblaciones con menores ingresos acuden menos a votar. Esta situación tiene carácter urbano y sería particularmente acentuada en las regiones Metropolitana, de Valparaíso, de Arica-Parinacota y de Los Ríos, lo que corresponde a cerca de la mitad de la población del territorio chileno. En definitiva, el mundo electoral en Chile ha tendido a despoblarse y, con ello, puesto en crisis los modelos de comprensión teórica y empírica de la democracia. Al respecto Contreras y Morales (2014, p. 612) concluyen que "la participación electoral es sólo una dimensión de la participación política". Esta es justamente la misma afirmación de Agamben (2010, p. 11) que plantea, a propósito del concepto de democracia, que en los debates actuales "el término se remite a la vez a la conceptualidad del derecho público y la de la práctica administrativa: designa tanto la forma de la legitimación del poder como las modalidades de su ejercicio". Se anuda aquí la trenza del misterio acerca de quién conduce la historia, pues se entrelazan el poder constituyente con el poder constituido en su inseparable relación con el poder soberano. Esta es la cartografía ideológica con sus puntos de referencia para adentrarse en la pregunta por el/la sujeto/a y su condición política: ¿qué tipo de pensamiento constituye y se constituye en el ejercicio del poder? ¿son las personas con menores ingresos aquellas que tienen mayor desapego al voto como expresión de ejercicio democrático?, es decir, ¿se trata de una voluntad colectiva? El problema no se puede restringir a la cuestión de qué significa el voto en la actualidad, sino que exige la apertura hacia comprender qué es lo político hoy, dónde se le encuentra y cómo se manifiesta. El Trabajo Social, como profesión orientada a la atención de las personas que viven situaciones de desigualdad o, directamente, de injusticia, requiere de una lectura que recupere la condición política del sujeto de intervención y que facilite la comprensión de la desvalorización del derecho político al voto que tan costoso le resultó a las generaciones anteriores durante los siglos 19 y 20. Esta conceptualización permite una interpretación de los datos y conduce a una propuesta de carácter académico para el ejercicio profesional; como también para algunos aspectos de la formación que habrían de considerarse como parte de los alcances que la intervención social debiera incluir como criterios de referencia para el reencantamiento de lo político del oficio y de los actores sociales implicados.

\section{Trabajo social y sujeto político: ruptura para entender la intervención social}

Los principios por los cuales el Trabajo Social define al sujeto de la intervención social son un tópico de la alta relevancia y complejidad (RUBILAR, 2013; ALAYÓN, 2005). Una breve puntualización es necesaria hacia la época de la modernidad con sus improntas de transformación social y de instalación del cogito como sujeto central de este proceso, es decir, el hombre y su razón eran los encargados de esta transformación social y el control de lo natural (CASTRO-SERRANO, 2012; MUÑOZ, 2011). Lo anterior impacta el devenir del mundo occidental, y por supuesto con implicancias para la disciplina del Trabajo Social en sus modos de explicarse la realidad. Dicho de otro modo, estos ribetes modernos cristalizan la manera de intervenir lo social a partir de un sujeto-identidad claro y preciso, que muchas veces se simplifica en su definición y en la manera 
de ser entendido para la intervención social. Esta última al comprender este tipo de subjetividad bajo esta constitución de sujeto, acciona desde marcos tecnocráticos y normativos que son más coercitivos que reparadores (CARBALLEDA, 2014; MATUS, 2004; MUÑOZ, 2014).

Aquí queremos pensar otra clave de lectura en perspectiva crítica, pues si toda intervención tiene riesgos de mecanizarse, tecnificarse y totalizarse a modo de control social, perdemos de vista el aparecer de ese otro que se interviene. La lectura en clave alteridad (LÉVINAS, 2006) nos pone de frente con aquella subjetividad que está ahí en esos otros que son parte de la intervención social, y eso nos permite re-leer al sujeto político que está en el ejercicio social. No implica una mirada totalizadora ni neutralizadora del otro, sino que amplía la mirada hacia la constitución de un sujeto fisurado y que ya no se cierra sobre sí mismo perdiendo ese lugar protagónico adquirido en la Modernidad. Estas cuestiones tienen implicancias hacia la intervención social en tanto puede abrirse al otro tomando una perspectiva de alteridad que hace reflexionar el quehacer del Trabajo Social (RUBILAR, 2013). Al reescribir la noción de sujeto como uno que está abierto a recibir al otro, se invierten los papales: el otro es anterior al sujeto lo que da cabida al acontecer intempestivo que trae el otro (LÉVINAS, 2006); nada comienza desde el sujeto totalizado, sino que se intenta "poner en el centro de los procesos de integración y de las prácticas de intervención a los propios excluidos, devolviéndoles su protagonismo restado, restaurando su presencia y fortaleciendo sus itinerarios de sentido" (RUBILAR, 2013, p. 59).

Entonces, cuando analizamos estas reflexiones en torno al tema de lo político y el voto, en el ámbito hispanoparlante no se ha encontrado una apreciación acerca de la condición de votante de las personas atendidas en los procesos de intervención social. Y aquí se despliega la veta para que el Trabajo Social pueda recomprender las formas de participación y ciudadanía. Es decir, al no clausurar el espacio político en el voto, es posible la aparición de un sujeto político que el TS no ha mirado. Las formas tradicionales, hegemónicas, naturalizadas de considerar a las personas en los procesos de intervención, fragmentan a un sujeto en categorías estáticas, predeterminadas y tecnificadas (MATUS, 2004), que cuando leemos este dato político en clave alteridad las posibilidades de intervención se amplían. Por lo tanto, el nuevo sujeto político existe antes de la intervención, ésta no lo determina, sino que debería asumirlo como un otro que participa y hace ciudadanía independiente de si vota o no, cuestión que se enmarca en los análisis que podemos realizar de la actualidad chilena. Frente a los antecedentes expuestos hasta aquí sobre las elecciones y la relación entre democracia y voto, la encuesta Ciudadanía hoy nos aporta empíricamente elementos para esta reflexión: se vuelve a reafirmar que la participación ciudadana no recae de modo exclusivo en el voto, y abre otros espacio respecto a las obligaciones ciudadanas como el pagar impuestos, obedecer las leyes y a las autoridades, entre otros.

Tabla 3 - "Cuáles de las siguientes acciones son para usted parte importante de las obligación de los ciudadanos: pagar honestamente los impuestos", de acuerdo a Grupo Socioeconómico (GSE) (en porcentaje).

\begin{tabular}{|l|c|c|c|c|c|}
\hline & GSE C1 & GSE C2 & GSE C3 & GSE DE & \multicolumn{2}{|c|}{ TOTAL } \\
\hline Sí & 13 & 24.1 & 34.8 & 28.2 & 100 \\
\hline No & 4.5 & 23.4 & 36 & 36 & 100 \\
\hline
\end{tabular}

Fuente: Elaboración propia.

Tabla 4 - "Cuáles de las siguientes acciones son para usted parte importante de las obligación de los ciudadanos: obedecer a las autoridades", de acuerdo a Grupo Socioeconómico (GSE) (en porcentaje)

\begin{tabular}{|l|c|c|c|c|c|}
\hline & GSE C1 & GSE C2 & GSE C3 & GSE DE & TOTAL \\
\hline Sí & 12.1 & 22.9 & 35.5 & 29.5 & 100 \\
\hline No & 13 & 30.2 & 31.8 & 25 & 100 \\
\hline
\end{tabular}

Fuente: Elaboración propia.

Los datos de las tablas 3 y 4 muestran cuestiones atractivas que reafirman nuestro recorrido: las obligaciones ciudadanas están en todos los grupos socio-económicos (GSE) repartidas de modo exacto 
respecto a la respuesta Sí y a la respuesta No, lo que podría indicar que hay una instancia más reflexiva y protagónica para comprender las obligaciones que tenemos como ciudadanos. No se deja ver una actuación desde el deber ser; y esto es muy interesante para ir reflexionado elementos de participación y ropajes políticos en los ciudadanos actuales, lo que a su vez, serían elementos interesantes a tener en cuenta en nuestras formulaciones de la intervención social. En este punto de nuestra reflexión surgen interrogantes atractivas para la disciplina nuestra: ¿cómo abordar este sujeto político desde otro lugar, o bien, desde otra manera de comprender la participación y la intervención social? En un paso más, ¿no será el Trabajo Social la opción disciplinar que puede hacerse cargo de este asunto?

Desde el propio campo disciplinar del Trabajo Social, pensando que su objeto de estudio es justamente el sujeto de la intervención social (MUÑOZ; VARGAS, 2013), estos datos nos indican que el sujeto político aquí pensado se configura bajo otras coordenadas; bajo otra lógica que renueva también las formas de intervención social en tanto nuevo sujeto/actor social, muchas veces desafectado del orden institucional, pero protagonista. Nuevo actor social que pujará los límites de la intervención social hacia su configuración precisa: como un espacio socio-político que no puede estar desafectado del lugar que ellos como 'otros' con rostros y un lugar preciso en su alteridad ocupan. Será un lugar a mirar en la intervención social para que realmente pueda ser una acción transformadora de la realidad social contemporánea (MUÑOZ,

2014). Este recorrido del sujeto político que fisura y abre nuevos espacios a la intervención social, nos obliga como disciplina a distinguir la política de lo político cuando intervenimos. Aquí puede ayudarnos la filosofía política en tanto la primera acepción nos indica las meras acciones políticas, partidistas e institucionales; pero la segunda, aquella de lo político, implica una chance de entender la cuestión política como la institución misma de lo social en toda democracia (ABENSOUR, 2009). Por tanto, creemos así que el Trabajo Social puede estar inserto desde esta mirada social-política para re-comprender los espacios de intervención, trabajando movilizados por el impacto de toda realidad injusta hacia el otro (RUBILAR, 2013).

En la misma senda, si continuamos sobrevolando los análisis del Abensour (2007), este nos ayuda instalando la necesidad de pensar la democracia y lo social como en una relación indisoluble y no en contraposición. Esto permite reflexionar una democracia constituida por los conflictos del mundo social y las subjetividades ahí instaladas, por tanto, está siempre excedida de sí misma y de su acontecer, no agotándose en la formalidad de la institución del voto y en lo agobiante del propio sistema neoliberal (CASTRO- SERRANO, 2015). Habrá que continuar reflexionando si estas articulaciones teóricas y empíricas pueden permear mayormente el Trabajo Social, para así poder estar constantemente repensando nuestro propio objeto de estudio: la intervención social (MUÑOZ; VARGAS, 2013).

\section{Conclusiones: comprensión de los contextos sociales}

El recorrido hecho en el presente artículo abre interesantes preguntas que desde el Trabajo Social constituyen un campo de posibilidades en el horizonte de comprender complejamente los escenarios contemporáneos de intervención. ¿Cuáles serán aquellos viejos espacios que el Trabajo Social debe resignificar para lograr la construcción socio-política de un nuevo sujeto en estos contextos de desigualdad ciudadana? ¿Cuáles son los anclajes teóricos y empíricos que la profesión debe mirar detenidamente para avanzar en ese sentido? El Trabajo Social debe intencionar una producción que densifique el conocimiento de los sectores populares, su imaginario y su práctica política. Conocemos poco acerca de los sujetos con/para quienes trabajamos y, en un contexto neoliberal como el chileno, colonizado por el mercado en esferas básicas para la calidad de vida, aquel conocimiento resulta central para que las intervenciones sociales provoquen movimientos contra hegemónicos. Las reflexiones que en este espacio se han expresado, toman el rumbo de la comprensión del nuevo sujeto político como motor de aquellos movimientos. En ese marco, una democracia de ciudadanos y para ciudadanos permite entender la relación entre la política y una recomprensión del Estado/Nación; el construir una nueva comunidad política para tomar decisiones y debatir hacia el consenso. Temas que habiendo estado tan ajenos a la reflexión del Trabajo Social, le son tan propios en su deseo de transformación social.

\section{Referencias}

ABENSOUR, M. Para una filosofía política crítica: Ensayos. Traducción de Scheherezade Pinilla y Jordi Riba. Barcelona: Anthropos, 2007. La Democracia contra el Estado. Traducción de Eduardo Rinesi. Buenos Aires: Coligue, 1998.

Pourquoi la Théorie critique? In: BENTOUHAMI, H., KUPIEC, A., GRANGÉ, N., SAADA, J. Le souci du droit, où en est la Théorie critique? Paris: Sens \& Tonka, 2009, p. 15-28. 
AGAMBEN, G. Nota preliminar sobre el concepto de la democracia. Traducción de Matthew Gajdowski In: AGAMBEN, G., BADIOU, A., BENSAÏD, D., BROWN, W., NANCY, J. L., RANCIÈRE, J., ROSS, K.; •I•EK, S. Democracia, ¿en qué estado? Buenos Aires: Prometeo libros, 2010, p. 11-13.

ALAYÓN, N. Trabajo social latinoamericano: a 40 años de la reconceptualización. Buenos Aires: Espacio Editorial, 2005.

ANGELCOS, N. Elementos para una crítica de la despolitización en Chile. Revista Observatorio de Juventud. Santiago de Chile: INJUV, 8, n. 29, p. 69-84, oct. 2011.

ARRIAGADA, E. Clientelismo político y participación local. Polis [En línea], n. 36, 2013. Disponible en: http://polis.revues.org/9389. Acceso en: 17 sept. 2015.

BAÑO, R. El nuevo carácter del apoliticismo. Serie Estudios Políticos. Santiago de Chile: Flacso, n. 33, p. 5-28, mar. 1995.

BLAIS, A. ¿Qué afecta a la participación electoral? Revista Española de Ciencia Política. Madrid: AECPA, n. 18, p. 9-27, Abr. 2008. CANTILLANA, C. Inscritos que no votan: la abstención electoral en Chile y sus factores explicativos. In: MORALES-QUIROGA, M., BRICEÑO R.; NAVIA P. El genoma electoral chileno. Dibujando el mapa genético de las preferencias políticas en Chile. Santiago: UDP, 2009, p. 77-96.

CARBALLEDA, A.J.M. La intervención social en los escenarios actuales: una mirada al contexto y el lazo social. Revista Intervención. Chile: UAH, n. 3, p. 57-60, sept. 2014.

CARLIN, R.E. The decline of citizen participation in electoral politics in post -authoritarian Chile. Democratization. UK: Routledge, v. 13, n. 4 , p. 632-651, ene. 2006.

CASTRO-SERRANO, B. Una fisura en la política clásica moderna abre la posibilidad de una nueva reflexión. Revista Iberofórum. México D.F.: UI, n. 13, p. 190-229, ene./jul. 2012.

El espacio de 'lo político' en Abensour como posibilitador de una 'democracia salvaje'. Revista Pensamiento. Madrid: UPC, v. 71, n. 265, p. 95-115, ene./abr. 2015.

CHIN, G.; CULOTTA, E. The science of inequality. What the numbers tell us. Science. USA: AAAS, v. 344, n. 6186, p. 818-821, may. 2014. DOI: $10.1126 /$ science.344.6186.818.

CONTRERAS-AGUIRRE, G.; MORALES-QUIROGA, M. Jóvenes y participación electoral en Chile 1989-2013. Analizando el efecto del voto voluntario. Revista Latinoamericana de Ciencias Sociales, Niñez y Juventud. Manizales: UC, v. 12, n. 2, p. 597-615, jul./ dic. 2014.

DAVIS, J. K. Voting as empowerment practice. American journal of psychiatric rehabilitation. UK: Routledge, v. 13, n. 4, p. 243-257, oct. 2010.

GARCÉS, M. Tomando su sitio: el movimiento de pobladores de Santiago, 1957-1970. Santiago de Chile: Lom, 2002.

GREZ, S. La ausencia de un poder constituyente democrático en la Historia de Chile. Tiempo histórico: revista de la Escuela de Historia. Santiago de Chile: UAHC, n. 1, p. 15-35, 2010.

ILLANES, M. A. Chile des-centrado: formación socio-cultural republicana y transición capitalista, 1810-1910. Santiago de Chile: Ed. Lom, 2003.

KIRKWOOD, J. Ser política en Chile. Las feministas y los partidos. Santiago: FLACSO, 1986.

LÉVINAS, E. Totalidad e infinito. Ensayo sobre la exterioridad. Traducción de Daniel E. Guillot. Salamanca: Ed. Sígueme, 2006.

MATUS, T. Propuestas contemporáneas en Trabajo Social. Hacia una intervención polifónica. Buenos Aires: Ed. Espacio, 2004.

MUÑOZ, G. Contrapuntos Epistemológicos para Intervenir lo Social: ¿Cómo impulsar un diálogo interdisciplinar? Cinta de Moebio. Chile: UCH, n. 40, p. 84-104, mar. 2011.

Aportes conceptuales y empíricos para pensar la intervención social interdisciplinaria en Chile. Revista Intervención. Chile: UAH, n. 3, p. 19-29, sept. 2014.

MUÑOZ, N.; VARGAS, P. A propósito de las tendencias epistemológicas de Trabajo Social

en el contexto latinoamericano. Revista Katálysis. Florianópolis: EDUFSC, v. 16, n. 1, p. 122-130, jan./jun. 2013.

OBSERVATORIO POLÍTICO-ELECTORAL (OBPE). Los ricos también votan. Participación electoral según nivel socioeconómico de las comunas en la Región Metropolitana. Santiago de Chile: ICSO/UDP, 2012.

OESCH, D. The changing shape of class voting. An individual-level analysis of party support in Britain, Germany and Switzerland. European Societies. UK: Routledge: vol. 10, n. 3, p. 329-355, ago. 2008.

PROGRAMA DE LAS NACIONES UNIDAD PARA EL DESARROLLO (PNUD). Auditoría a la democracia. Más y mejor democracia para un Chile inclusivo. Santiago de Chile: PNUD, 2014.

La democracia en América Latina: hacia una democracia de ciudadanas y ciudadanos. Buenos Aires: Aguilar, Altea, Taurus, Alfaguara, 2004.

RUBILAR, G. Imágenes de Alteridad. Reflexiones y aportes para el trabajo social en contextos de pobreza y exclusión. Santiago de Chile: Ed. UC, 2013.

TOGUCHI, T. et al. Welfare and Citizenship: The Effects of Government Assistance on Young Adults' Civic Participation, The Sociological Quarterly. UK: n. 50, p. 633-665, oct. 2009.

UNIVERSIDAD ANDRÉS BELLO-UNAB. Encuesta Ciudadanía hoy. Santiago de Chile: Facultad de Humanidades y Ciencias Sociales, 2012.

VITALE, L. La mitad invisible de la historia. El protagonismo social de la mujer latinoamericana. Buenos Aires: Sudamericana/Planeta, 1987. 


\section{Notas}

1 Otros autores que aportaron en esta investigación: Marcela Flotts, Universidad Andres Bello, Chile; Jorge Jofré, Universidad Andres Bello, Chile.

2 No obstante, la universalidad debe matizarse en tanto las legislaciones electorales siempre administran el acceso de los grupos con capacidad de goce del derecho a voto.

3 Ficha técnica: universo: población de 16 años y más, residente en núcleos urbanos de más de 100 mil habitantes, y en las ciudades de Coyhaique y Aysén. Tamaño muestral: se entrevistó cara a cara a 1.300 personas a lo largo de todo el país. Diseño muestral: se efectuó un muestreo probabilístico trietápico no proporcional. Las unidades de las tres etapas de muestreo son las siguientes: selección aleatoria de manzanas en los núcleos urbanos bajo estudio; selección aleatoria de hogares en cada manzana sorteada, y selección aleatoria de individuos en cada hogar. Nivel de precisión: el error muestral se estima en 3\%, considerando el supuesto de varianza máxima y 95\% de confianza. Instrumento: se utilizó un cuestionario semiestructurado con una duración de 20 a 25 minutos. Fecha de terreno: el trabajo de campo se ejecutó en octubre de 2012 (UNAB, 2012).

\section{Nelson Arellano-Escudero}

narellano.5@gmail.com

Doctor en Sustentabilidad, tecnología y humanismo por la Universidad Politécnica de Cataluña (Barcelona, España)

Profesor de la Escuela de Trabajo Social, Universidad Andres Bello (UNAB), Chile.

\section{Borja Castro-Serrano}

francisco.castro@unab.cl

Doctor en Filosofía por la Universidad de Murcia (España).

Profesor de la Escuela de Trabajo Social, Universidad Andres Bello (UNAB), Chile.

\section{Marcela Flotts de los Hoyos}

mflotts@unab.cl

Magíster en Desarrollo Humano por la Universidad La Frontera (Chile)

Es directora de la Escuela de Trabajo Social, Universidad Andres Bello (UNAB), Chile.

\section{Jorge Jofre Alfaro}

jorge.jofre@unab.cl

Magíster en Políticas Sociales por la Universidad de Artes y Ciencias Sociales (ARCIS).

Profesor Escuela de Trabajo Social Universidad Andrés Bello (UNAB).

\section{UNAB}

Facultad de Humanidades y Ciencias Sociales

República $\mathrm{N}^{\circ} 252$

Santiago - Chile

Código Postal: 8370134 